\title{
TSH IN THE UPPER LIMITS OF THE NORMAL RANGE IS ASSOCIATED WITH AN ADVERSE LIPID PROFILE IN EUTHYROID NON-DIABETICS WITH NEWLY DIAGNOSED ASYMPTOMATIC CORONARY HEART DISEASE
}

doi:10.1136/heartjnl-2012-302920j.9

Xing Wanjia, Zhao Jiajun. Provincial Hospital Affiliated to Shandong University

Objectives Lipid profile is influenced by both thyroid function and glucose metabolism. Increasing evidence demonstrated that TSH might affect the cardiovascular risk factors through some extrathyroidal effects. The goal of this study was to evaluate the relationship between serum TSH levels and the lipid profile in euthyroid non-diabetics with newly diagnosed asymptomatic coronary heart disease (CHD).

Methods A retrospective study was conducted including 438 euthyroid non-diabetics (209 males and 229 females, age ranged 40-88 years) with newly diagnosed asymptomatic CHD from 2004 to 2010 in Jinan, China. Lipid parameters and the levels of TSH, thyroid hormones were determined. The patients were divided into three groups according to different TSH levels within the normal range: a lower limits TSH subgroup (TSH 0.3-0.99 mIU/l, G1), a moderate TSH subgroup (TSH 1.0-1.89 mIU/l, G2) and a upper limits TSH subgroup (TSH 1.9-4.8 mIU/l, G3). Regression analysis and ORs were used to assess the influence of the parameters on the lipid profile and to estimate the risks of dyslipidemia with respect to the TSH levels. 
Results The TSH level, even within the normal range, was positively and linearly correlated with total cholesterol (TC), non-high density lipoprotein cholesterol (non-HDL-C) and triglycerides (TG) $(\beta=0.200$, 0.224 and 0.128 , respectively; $p<0.001, p<0.001$ and $p<0.05$, respectively). Within the normal range, the ORs of the risk of hypercholesterolaemia and hypertriglyceridaemia with respect to TSH level within normal range were 1.927 (95\% CI 1.449 to 2.561, p<0.001) and 1.266 (95\% CI 1.013 to $1.584, p=0.038$ ), respectively. When running subgroup analysis, compared with those in G1, the TC levels were significantly higher in G2 $(5.10 \pm 1.04$ vs $4.79 \pm 1.01 \mathrm{mmol} / \mathrm{l}, \mathrm{p}<0.01)$ and G3 $(5.20 \pm 1.02$ vs $4.79 \pm 1.01 \mathrm{mmol} / \mathrm{l}, \mathrm{p}<0.01)$. The levels of LDL-C were significantly higher in G2 and G3 when compared with those in G1 $(3.13 \pm 0.79$ vs $2.84 \pm 0.65 \mathrm{mmol} / 1,3.06 \pm 0.78$ vs $2.84 \pm 0.65 \mathrm{mmol} / \mathrm{l}$, respectively, $p<0.01$ and $p<0.05$, respectively). The levels of nonHDL-C were also significantly higher in G2 and G3 when compared with those in G1 $(3.83 \pm 0.98$ vs $3.46 \pm 1.02 \mathrm{mmol} / 1,3.89 \pm 1.07$ vs 3.46 $\pm 1.02 \mathrm{mmol} / \mathrm{l}$, respectively, $\mathrm{p}<0.01$ in both). The prevalence of hypercholesterolaemia in G2 and G3 was significantly higher than that in G1 $(16.78 \%$ vs $4.05 \%, 18.60 \%$ vs $4.05 \%$, respectively; $p<0.01$ in both). For hypertriglyceridaemia, the prevalence was approximately 2-folds greater in G2 and G3 than that in G1 (34.90\% vs $17.57 \%$, $36.28 \%$ vs $17.57 \%$, respectively; $\mathrm{p}<0.01$ in both).

Conclusions TSH levels were associated in a positive and linear manner with the TC, non-HDL-C and TG levels in euthyroid non-diabetics with newly diagnosed asymptomatic CHD. TSH in the upper limits of normal range might exert adverse effects on the lipid profile and might represent a risk factor for hypercholesterolaemia and hypertriglyceridaemia in the context of CHD.

Maintaining TSH in a relative low normal range might be beneficial for lipid profile in euthyroid non-diabetics with newly diagnosed asymptomatic CHD. 